\title{
A potential new strategy for using elastase and its inhibitor as therapeutic agents
}

\author{
Tarhini $M^{1,2}$, Fessi $\mathbf{H}^{1}$, Bentaher $A^{3}$, Greige-Gerges $\mathbf{H}^{2}$ and Elaissari $A^{1 *}$ \\ ${ }^{1}$ University Lyon, Universite Claude Bernard Lyon-1, CNRS, LAGEP UMR 5007, 43 boulevard du 11 Novembre 1918, F-69100, Villeurbanne, France \\ ${ }^{2}$ Faculty of Sciences, Lebanese University, B.P. 90656, Jdaidet El-Matn, Lebanon \\ ${ }^{3}$ Inflammation and Immunity of the Respiratory Epithelium - EA 7426, Faculte de Medecine Lyon Sud, 69495, Pierre Benite, France
}

\begin{abstract}
Human neutrophil elastase is a proteolytic enzyme secreted by neutrophil during inflammation and infection in lung environment. It has an important anti-bacterial activity through different pathways. However, it also has an important proteolytic action against lung's extracellular matrix which lead to different disease related to acute lung injuries. secretory leukocyte proteinase inhibitor (SLPI) is a naturally produced protein with an elastase inhibition main role. Different lung diseases were linked to the imbalance of elastase/SLPI ratio in lung environment. In addition, SLPI has an important antibacterial activity. In this review, a new strategy was suggested to use elastase and SLPI as therapeutic agents. Using encapsulation technology, the entrapment of these molecules inside albumin nanoparticles could produce a great opportunity in tissue degradation protection and non-antibiotic related bacterial killing.
\end{abstract}

Graphical abstract: The application of NE and SLPI loaded nanoparticles in antibacterial killing and lung tissue protection (Figure 1).

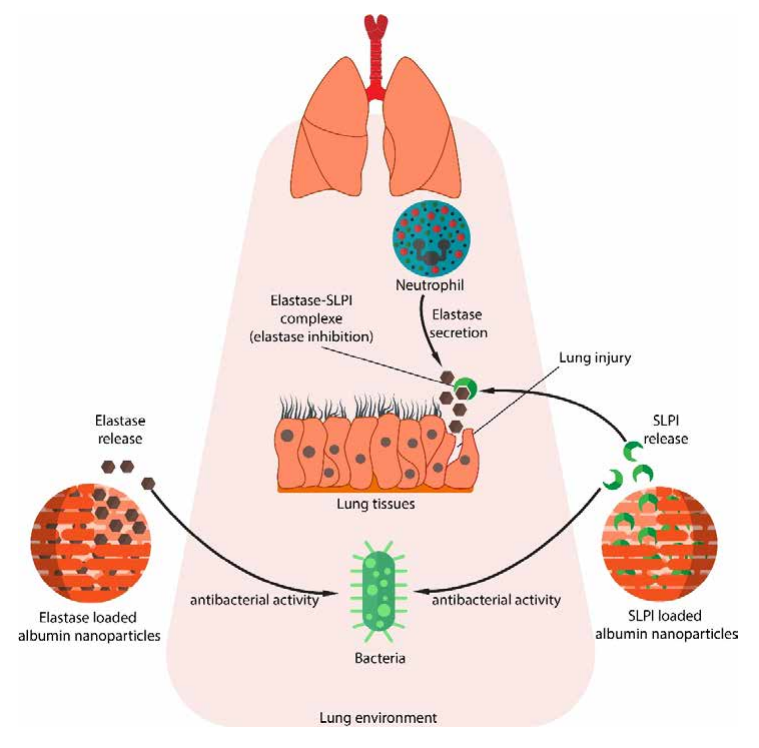

\section{Introduction}

The immune system is complex enough to give the body the ability to efficiently fight against a wide range of diseases, infections, etc... After biological barriers, the first line of host defense against infection is the recruitment of neutrophils to inflammation sites [1]. Neutrophils are granulated polymorphonuclear leukocytes that eliminates the foreign microorganisms through phagocytosis. These major players are known to contain four types of granules: azurophil, specific granules, gelatinase granules, and secretory granules. While each type of granule has certain role according to their content and their behavior during infection, azurophil granules (also known as primary granules) are heterogeneous in size and have different morphological features. They are packaged with acidic hydrolases and antimicrobial proteins. In addition, azurophils contain three structurally related enzymes: human neutrophil elastase (NE), cathepsin G (CG), and protease-3 (PR3) [2]. From these three enzymes, HNE is the product of interest in this review.

${ }^{\star}$ Correspondence to: Abdelhamid Elaissari, University Lyon, Universite Claude Bernard Lyon-1, CNRS, LAGEP UMR 5007, 43 boulevard du 11 Novembre 1918, F-69100, Villeurbanne, France, E-mail: abdelhamid.elaissari@univ-lyon1.fr

Key words: elastase, antibacterial, nanoparticles, albumin, secretory leukocyte protease inhibitor

Received: October 29, 2018; Accepted: November 09, 2018; Published: November 12, 2018 
$\mathrm{NE}$ is a serine protease of the chymotrypsin family. It is known as degradative enzyme playing an important role in the elimination of the extracellular pathogens and breaking down tissues at inflammation sites. It is also being identified as molecular targets for anti-inflammatory agents $[3,4]$. The biosynthesis of NE has two regulation point, the first one is at the transcriptional level. Genes of NE are heavily regulated and expressed during cell development specifically during promyelocytic phase (during myeloid development). NE gene expression will then be downregulated with the maturation of neutrophil. This is the reason NE only exist in the azurophil granules of neutrophil. Since the localization of protein does not depends on its own individual sorting information but on the time of its biosynthesis, which is the same time of azurophil formation [5]. The second regulation point is post-translational, where the inactive form of $\mathrm{NE}$ gone with different modification to became active. NE is synthetized as an inactive prepro-protein containing a signal peptide, $\mathrm{N}$-terminal prodipeptide, and a C-terminal propeptide. For NE to be activated, a cleavage of the signal peptide by signal peptidase must be occur. After that, the $\mathrm{N}$-terminal dipeptide must be removed by DPPI (also known as cathepsin $\mathrm{C}$ and widely expressed in myeloid cells) (Figure 2). These modifications lead to the formation of a fully mature active NE with 218 amino acid residue and a mass of 29 to $34 \mathrm{KDa}[6,7]$.

The mature human neutrophil elastase is said to be containing 218 amino acid residues and constitute of a single polypeptide chain. It also has four intra-chain disulfide bridges, two asparagine-linked carbohydrate chains, no lysine or tyrosine residues which make it extremely cationic, and 19 arginyl residues. These compositions give the protein a very high isoelectric point $(10-10.5)[3,8]$. The active site of mature NE can be described with as shown in Figure 2. The different $S$ pockets in the enzyme's active site are the docking sites of $P$ residues in the substrate's cleavage site. The cleave of the substrate take place between $\mathrm{P} 1$ and $\mathrm{P} 1$ ' residues. The $\mathrm{S} 1$ pocket of the active site is known to be hydrophobic due to the presence of Val190, Phe192, Ala213, Val216, Phe228, and a disulfide bridge at Cys191-Cys220. Therefore, small hydrophobic residues are needed in the P1 site of the substrate such as Val, Cys, Ala, Met, or Ile in order to achieve an enzymatic activity $[9,10]$. The S2 pocket of the enzyme contains Phe215 and Leu99 and the flat side of the imidazole ring of the catalytic triad His57, which give this site a hydrophobic nature. This hydrophobic nature of S2 give the preference of medium hydrophobic side chains (such as Pro) to be in the P2 site of the substrate. As for S1' and S2' pockets, they are also hydrophobic due to the presence of the disulfide bridge Cys42-Cys58 and Phe41, Leu143 respectively in these pockets [11] (Figure 3).

\section{Biological activity of human neutrophil elastase}

\section{Antimicrobial effect}

$\mathrm{NE}$ is known as multifunctional enzymes with an activity spectrum ranging from its involvement in pathogen killing to its regulatory role in the inflammation process [12]. The microbial killing activity of NE can be seen intracellular on phagocytosed bacteria in phagolysosomes [13], or extracellular by forming trap networks where the bacteria can be trapped [14]. It was found the NE is the key factor in preventing Shigella (Gram-negative bacteria) from escaping neutrophil's phagocytic vacuoles. In addition, NE showed to be able to degrade Shigella virulence factors (IpA, B, C, and IcsA) at a 1000fold lower concentration than that needed to degrade other bacterial proteins. These finding is supported by the fact that in genetically or pharmacologically inactive NE neutrophils, the bacterial survival increase by increasing its chances to escape form phagosomes [15]. In

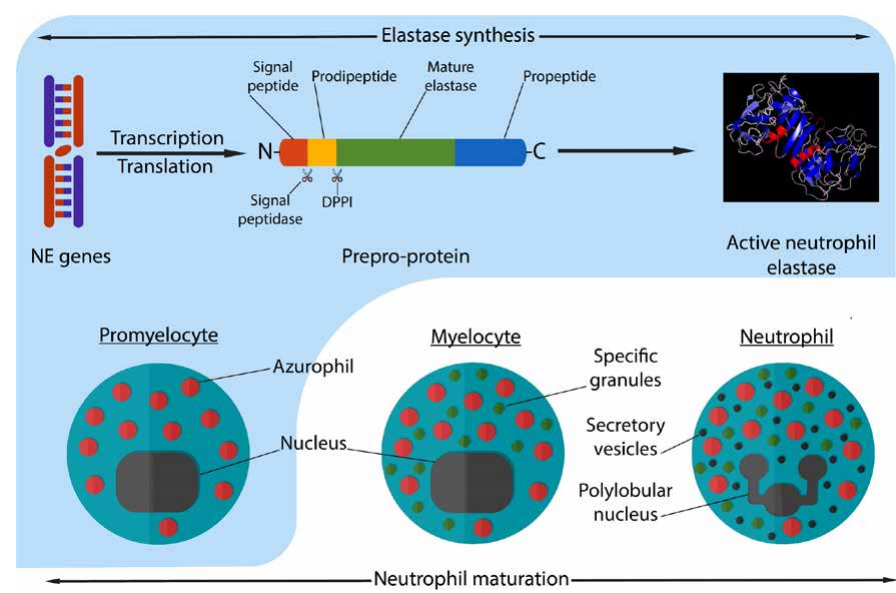

Figure 2. Neutrophil maturation process, granules synthesis at each phase and synthesis of active neutrophil elastase during these phases. The formation of azurophil and the transcription-translation of elastase genes occurs during promyelocytic stage of neutrophil maturation

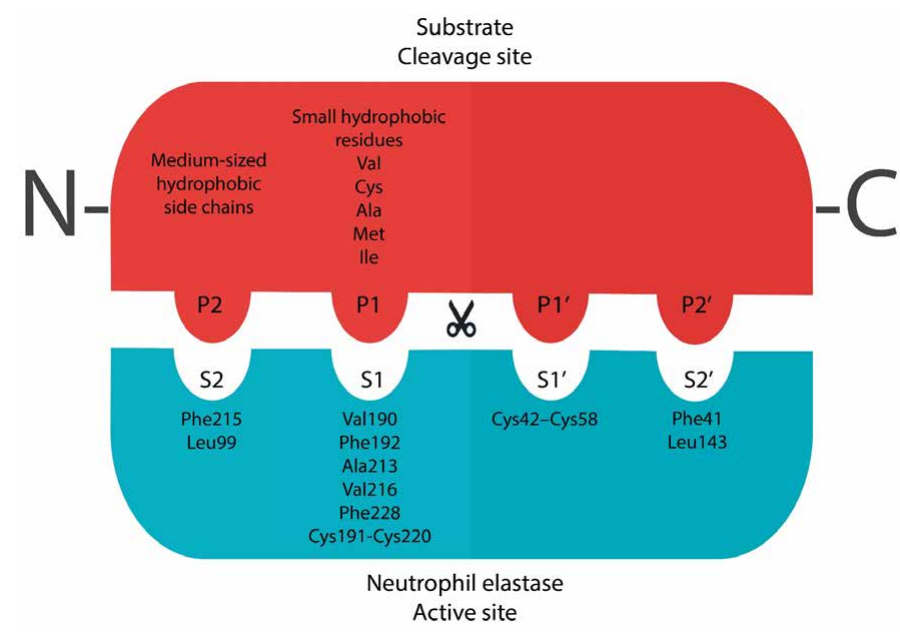

Figure 3. The active site of NE mature enzyme and the cleavage site of a substrate

other study on Escherichia coli, NE was confirmed to be localized in phagolysosomes following the ingestion of the bacteria by neutrophil. By investigating NE's mechanism of bacterial killing, it was found that the catalytic activity of NE is responsible for the degradation of the bacterial outer membrane protein A (OmpA) (gram-negative exclusive membrane). In this study, NE was proven to be a direct factor in non-oxidative bacterial killing pathways of neutrophil [16] (Figure $4 \mathrm{~A})$. NE was also found to be important in the oxygen dependent antimicrobial system for both gram positive and negative bacteria. In a study made on Escherichia coli (Gram negative) and Staphylococcus aureus (Gram positive), it was observed that the antibacterial activity of myeloperoxidase (MPO)-glucose oxidase system increases in the presence of NE. The killing of both bacteria was done at an acidic $\mathrm{pH}$, and at $\mathrm{pH} 7.4$ only killing of E.coli was potentiated. In addition, it was found that the potentiating effect of elastase was not dependent on the enzymatic properties of elastase since it was not reduced by heating, suggesting that the effect of elastase possibly involve the interaction between the cationic enzyme and the negatively charged bacteria [17] (Figure 4B).

NE also have an extracellular activity. Upon activation, neutrophils release its granule proteins (including NE) and chromatin that together form what so called neutrophil extracellular traps (NETs). These 
traps are known to bind to gram-negative and gram-positive bacteria, prevent them from spreading, and ensure a high local concentration of antimicrobial agent [18]. The formation of NETs is accompanied by the loss of neutrophil's nuclear lobules and the decondensation of chromatin, detachment of the inner nuclear membrane from the outer one, and disintegration of the granules. NETs are formed by disintegration of the nuclear envelope and release of the cellular content into the extracellular space. In this phenomenon, NE plays a role in the degradation of $\mathrm{H} 1$ linker histone and lead to the decondensation of chromatin. In addition, NE is present in NET with other components as an antimicrobial agent (serine proteases and MPO) [19]. In a study performed on a Papillion-Lefever syndrome patient (results from mutations that inactivates cysteine protease cathepsin $C$ that is necessary to process a variety of serine protease including NE). The inactivation of DPPI cause in addition to the lack of NE, the inability to generate NETs. Another study showed that NE knockout mice do not NETs in a pulmonary model of Klebsuekka pneumonia infection. Results from these studies support the claim that NE participate in NET formation by promoting chromatin condensation [20,21] (Figure 4C).

\section{Inflammation regulation effect}

In addition to the antibacterial role, NE are important in the regulation of the local inflammatory responses. NE can have control of cellular signaling through the processing of chemokines, modulating the cytokine network and activating specific cell surface receptors [22]. IL-1 $\beta$ is an inflammatory cytokine synthetized as inactive membrane bound form and became active by the cysteine protease IL-1 $\beta$ converting enzyme (caspase-1). It was found that the release of active IL- $1 \beta$ was attenuated significantly by inhibition of neutrophil elastase. In addition, it was found that NE cleaves the pro-isoform of IL- $1 \beta$ in endothelial cells and lead to a significant secretion of active IL- $1 \beta$ via extracellular vesicles, shedding the light on a potential antiIL1 $\beta$ therapeutic strategy [23]. In other study, the effect of NE on IL36 receptor antagonist (IL-36Ra) was investigated. IL-36 cytokines are members of IL-1 super-family and consisting of agonists IL-36a, IL-
$36 \beta$, and IL-36 $\gamma$, and antagonist IL-36Ra. The agonist binds to a specific receptor IL-36R causing a series of events that leads to the activation of the nuclear factor NF- $\mathrm{KB}$ and therefore the expression of a host of proinflammatory mediators. However, the antagonist binds to the same receptor without leading to the recruitment of the accessory proteins and inhibits the activity of IL-36 cytokines. NE was studied in human primary dermal fibroblasts and keratinocytes and in skin equivalents, it was found that NE cleaves IL-36Ra into its highly active antagonist form. Compared to the full length IL36-Ra, NE cleaved antagonist inhibit IL-36 induced chemokine production and reduce further inflammatory cell infiltration [24].

$\mathrm{NE}$ also plays a role in the regulation of growth factors. NE is known to modulate the cleavage of the membrane-bound transforming growth factor alpha (TGF- $\alpha$ ), releasing a soluble form that can bind to and activate the epidermal growth factor receptor (EGFR). This receptor has been shown to be a major cause of mucus secretion. The activation of EGFR promotes mucous cell hyperplasia and MUC5AC mRNA expression $[25,26]$. Moreover, TGF- $\alpha$ liberated by NE induces keratinocyte proliferation via EGFR activation [27]. In addition, NE also activates the transforming growth factor TGF- $\beta$. The latent form of this growth factor is composed of the mature TGF- $\beta$ homodimer, the latency-associated protein, and the latent TGF- $\beta$-binding protein (LTBP). The proteolysis of LTBP by NE release TGF- $\beta$ from the extracellular matrix and therefore contributes to the tissue remodeling that accompanies inflammation in the lungs [28].

\section{Neutrophil elastase related pathology}

The accumulation of neutrophils in the microvasculature of the lung is a major hallmark of chronic inflammatory lung diseases. The inflammatory responses are can sometimes results in a further organ injury and dysfunction [7]. In fact, different studies were able to relate NE to the development of chronic inflammatory lung diseases $[29,30]$.

Chronic obstructive pulmonary disease (COPD), is an advanced inflammatory response of lungs parenchyma against harmful particles

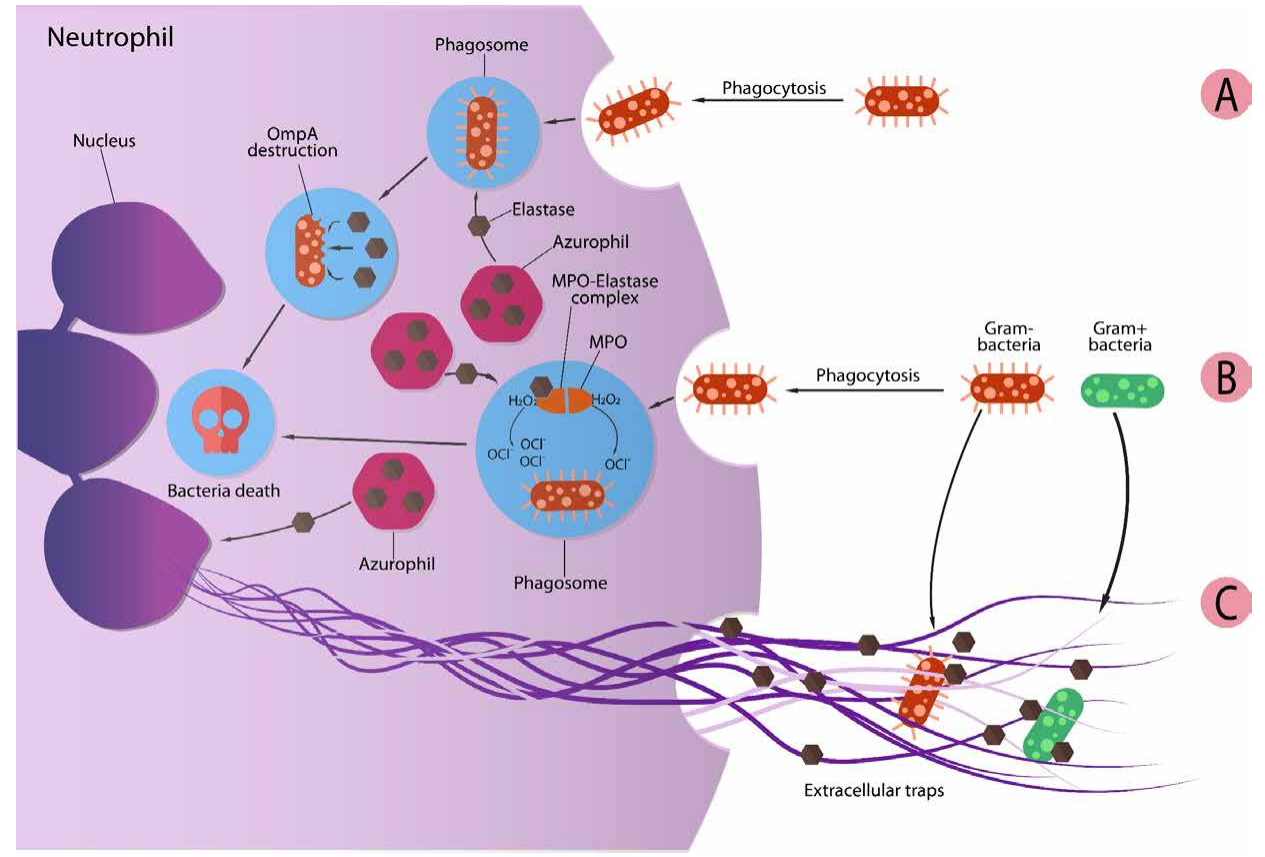

Figure 4. Antibacterial activity of NE: A) destruction of bacteria by direct interference of NE. B) cooperation between NE and MPO for bacteria killing. And C) contribution of NE in extracellular traps formation 
and gases. The obstruction of airways is due to the loss of alveolar attachments, and mucosal inflammation. This phenomenon can cause structural damage, airways narrowing, and lung parenchyma destructions [31]. Different studies have linked NE activity with COPD. In a study made on human bronchial epithelial cells BEAS2B exposed to cigarette smoke extracts (CSE), a cooperation between $\mathrm{NE}$ and CSE was observed [32]. CSE increase the production of both interleukin-8 (IL-8), and vascular endothelial growth factor (VEGF) in the used cells. NE was found to significantly enhance CSE-induced IL-8 production, while suppressing VEGF production. IL-8 plays a role in neutrophil recruitment and VEGF Stimulates endothelial cell and type II epithelial cell growth and survival. In fact, the loss of VEGF can lead to apoptosis of epithelial cells in the emphysematous lung [33]. In contrast, considering the actions of IL-8 and VEGF, NE contribute to the pathogenesis of COPD by enhancing inflammation and apoptosis [30].

NE also was found to promote acute lung injury (ALI). This syndrome is caused by the infection of Gram-negative bacteria, and its pathophysiological characteristics include interstitial and alveolar edema and infiltration of these spaces by many neutrophils. The most dangerous version of this syndrome, adult respiratory distress syndrome (ARDS), is considered a high mortality disease and have the ability to resist many therapies [10]. NE is considered a powerful host defense and its activity is regulated by various endogenous protease inhibitors. However, at inflammatory sites, an imbalance between NE and its inhibitor lead to a continuous activity of NE caused by and elevation in its level. This unregulated activity can lead to different changes that are linked to pathophysiology of acute injury in lungs [34,35]. A recent study showed that NE contribute to degradation of epithelial cadherin (EC) in inflamed lung situation. EC is known to reinforce the stability of the epithelium. In addition, it has a role in molecular signaling and epithelium permeability regulation. This study shows that as long as significant amounts of active NE are available, the degradation of EC could be observed, suggesting that NE interfere with cell-cell adhesion function, and participate in neutrophil-rich lung inflammatory and tissue-destructive diseases [36].

\section{Inhibition of neutrophil elastase}

The activity of elastase can be reduced by different endogenous inhibitors such as $\alpha-1$ proteinase inhibitor, human monocyte / neutrophil elastase inhibitor, and secretory leukocyte proteinase inhibitor (SLPI), the product of interest [37].

SLPI is a non-glycosylated protein found in all body fluids such as tears, salivary glands, seminal fluids, bronchial secretions, cervical and intestinal mucus. It is a relatively small protein composed of 107 amino acids and $11.7 \mathrm{KDa}$. Is highly basic with an isoelectric point $\mathrm{pI}>11$ [38]. SLPI is known to be a reversible inhibitor for NE, chymotrypsin, chymase, and bovine trypsin. It possesses to homologous whey acidic proteins (WAP) domains in which eight cysteine residues are present in each one that forms eight intramolecular disulfide bonds. The inhibitory function of SLPI against NE is caused by the WAP2 domain of SLPI. The inhibitory region is localized between residues 67 and 74 of SLPI sequence (Figure 5A). Koizumi et al. have crystallized a complex of NE and the WAP2 domain of SLPI. The X-ray data showed the importance of the residues L72 and Y68 in the primary contact regions for inhibiting NE by forming hydrogen bonds with it [37] (Figure 5B).

SLPI is known to be a reversible inhibitor of NE with association constant K_ass $=6.4 \times[10] \wedge 6 \quad \mathrm{M}^{\wedge}(-1) . \mathrm{s}^{\wedge}(-1)$, dissociation constant K_diss $=2.3 \times[10] \wedge(-3) \mathrm{s}^{\wedge}(-1)$, and inhibition constant $\mathrm{K} \_\mathrm{i}=3 \times[10] \wedge(-$

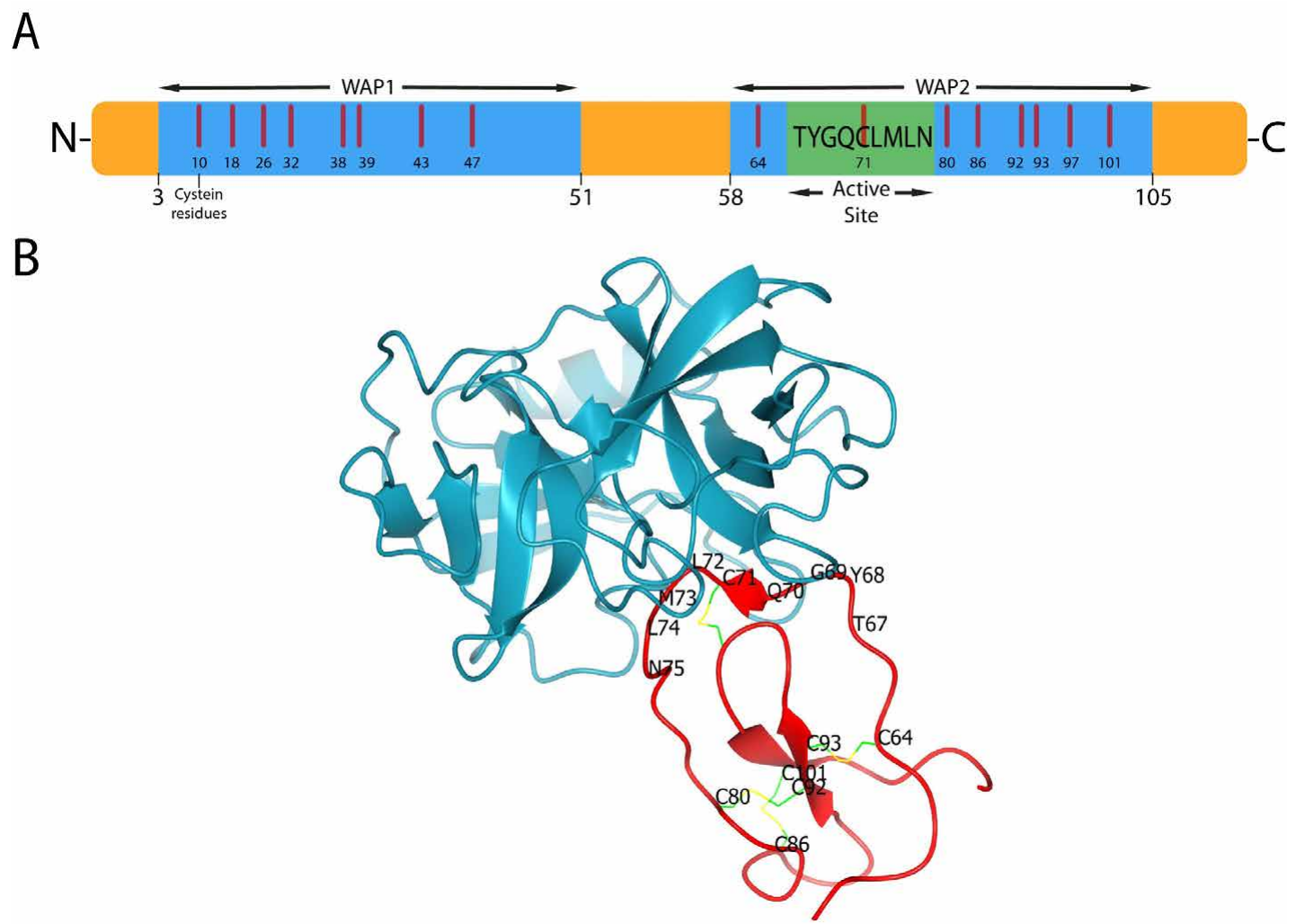

Figure 5. (A) SLPI structure and interaction A) the different domains of SLPI, (B) The interaction of WAP2 domain with neutrophil elastase. Amino acid residues of the active site and disulfide bonds are shown. PDB ID: 2Z7F 
10) $M$ [39]. It accounts for $80-95 \%$ of the NE inhibitory capacity of human upper respiratory tract secretion [40]. The inhibition activity of SLPI towards NE can also reach the extracellular matrix. Due to the low molecular weight of SLPI, it can protect the extracellular matrix proteins from proteolysis by $\mathrm{NE}$ by accessing the space between adherent neutrophil and the matrix [41]. In additon, SLPI have an inhibitory effect on the formation of NET. Since NE is an important factor in the NET formation, its inhibition by SLPI can regulate NET release in vitro and in vivo. However, the use of an active site mutated SLPI partially restrict NET formation, this can signify that SLPI can control NET formation by targeting proteases other than NE [42].

\section{SLPI beyond the inhibition of elastase}

Despite the inhibition of human neutrophil elastase being the major role of SLPI, research suggests other functions of this relatively small protein such as anti-inflammatory, anti-bacterial, and anti-viral $[43,44]$.

\section{Anti-inflammatory role}

SLPI was proven to prevent the activation of nuclear factor $\kappa B$ (NF- $\mathrm{kB}$ ) which is a transcription factor related to the increase of pro-inflammatory mediators during lung inflammation, and recently demonstrated, in acute liver failure [45]. This activity is clearly beyond the NE and other protease inhibition activity of SLPI since the use of active site-mutated SLPI can inhibit NF- $\mathrm{BB}$ by preventing the degradation of its inhibitory components such as I $\mathrm{I} B \alpha$ and I $\kappa \mathrm{B} \beta$. This behavior leads to the attenuation of the macrophage response to lipopolysaccharides and prevent NF- $\mathrm{KB}$ from entering the nucleus and activate the transcription of pro-inflammatory genes [46]. In addition, SLPI interfere with NF- $\mathrm{BB}$ cascade in the nucleus. In a study on U937 monocytic cell lines, it was found that upon incubation, SLPI rapidly localize in cytoplasm and nucleus, where it compete with p65 for binding to the NF- $\mathrm{kB}$ binding sites in the promoter region of proinflammatory genes like IL-8 and TNF- $\alpha$ [47].

\section{Antimicrobial role}

The antibacterial activity of SLPI covers the gram negative and gram positive bacteria such as Escherichia coli, Pseudomonas aeruginosa, Staphylococcus aureus, and Staphylococcus epidermidis. This activity has beed atributed mainly to the non-inhibitory $\mathrm{N}$-terminal WAP1 domain of SLPI. In fact, the median effective dose (ED50) of the N-terminal domain of SLPI was 12.1 and $31.2 \mu \mathrm{M}$ for E. coli and S. aureus respectively. However, the C-terminal domain also shows a relatively low antibacterial activity with ED50 of $23.7 \mu \mathrm{M}$ on E. coli, and undetermined on S. aureus. But the use of a full intact SLPI shows the lowest ED50 for both bacteria (4.2 and 7.6 $\mu \mathrm{M}$ for E. coli and S. aureus respectively) [48].

Different mechanism where suggested to explain the antibacterial effect of SLPI. Miller et al. demonstrated that when SLPI is overexpressed in E.coli, it can bind to DNA and mRNA and inhibit transcription and translation. It was suggested that SLPI can bind to the bacterial mRNA and DNA via electrostatic interactions and blocks the protein synthesis, and therefore causes bacterial toxicity [49]. Another mecanism was suggested related to the high psitive charge of SLPI WAP domains. Where this positive charge could induce interactions with anionic bacterial lipids, which lead to the disruption of the bacterial membrane by SLPI. This claim can be supported by the fact that WAP1 domain that have a higher positive charge $(+7)$ than WAP2 domain $(+5)$, also have a higher antimicrobial activity [50]. Moreover, it was found that higher salt concentration could inhibit the antibacterial activity of SLPI [47].

SLPI also have an antviral role, it was indentified as the main factor of anit-HIV inhibition activity in human saliva [51]. In addition, the increase of SLPI concentration in vaginal fluids was associated with significantly reduced rates of perinatal HIV-1 transmission [52].

\section{Therapeutic strategies of NE and SLPI}

Different strategies were tested in the literature to overcome elastase related inflammatory in infectious lung diseases. For example, the administration of therapeutic inhibitors of NE to control the unwanted proteolysis in COPD [53]. Different inhibitors were suggested and administrated via different routes (oral, intravenous, by an aerosol route). However, these inhibitors always suffer from the limitation of physicochemical constraints and the fact that the protease exist in soluble and solid phases such as NET. In addition, enzyme inhibitors are generally hydrophobic, while the lungs are highly perfused, this results in limited efficacy towards NE due to high clearance [54]. To overcome this limitation, a study linked covalently an elastase inhibitor to a 25 -amino acid fragment of human surfactant peptide $B$. this gives the complex a long lung residence time and prevented NE-induced emphysema in a rodent model [55]. Despite the presence of a wide range on NE inhibitors, only few have been validated for therapeutic use in clinical settings. In fact, Sivelestat is the only synthetic NE inhibitor in market and approved by Japan and South Korea. Sivelestat have been suggested as a treatment for acute lung injury and acute respiratory distress syndrome [56]. However, in case of COPD, its complexity may require the administration of more than one agent with different functions, or a dual function agent able of reducing proteolysis and inflammation $[57,58]$.

As shown above, elastase has an important antibacterial role and can overcome infection threw different mechanism. However, NE was not used as an administrated antibacterial agent due to its important proteolysis function. The in-vitro success of NE against bacteria can be translated to in-vivo administration if, in theory, NE was able to specifically target bacteria without having a huge effect on the related environment. To our knowledge, no previous research has been focused on this matter. However, different drug targeting techniques were applied for different kind of bioactive molecules and macromolecules in order to increase the efficiency of the drug and reduce its side effect [59]. Form these techniques, the use of a drug carrier to entrap the active molecule was proven to be a very successful strategy.

\section{Nanoparticles as drug carrier}

Nanoparticles have become a major player in the encapsulation field, the use of a natural or synthetic polymer in order to produce nano-sized carrier was applied in the industrial, agricultural, and pharmaceutical domain [60]. However, for in-vivo administration, the used polymer should have a certain criterion to be usable. It should be non-toxic at the first place and biodegradable so it does not leave traces of undesirable side products [61]. The importance of nanoparticles comes from the fact that by entrapping a certain molecule inside, its circulation will be facilitated, this is also noticeable when encapsulating hydrophobic compounds. In addition, the release of this compound in the target site will be continuous and controllable, and also specific, where side effects of a certain compound could be removed [62].

From a wide range of polymers chosen to prepare nanoparticles, proteins may be the best candidate. They are natural and widely available, non-toxic, biodegradable, and does not leave undesirable 
by-products since they are composed of amino acids. In addition, the presence of a wide range of proteins with different physicochemical characteristics allows this technology to be successful to encapsulate a wide range of active compounds of different types (hydrophilic, hydrophobic, macromolecules, etc.). Table 1 showed an example of different kinds of proteins used to prepare nanoparticles as carriers for many types of active compounds.

Among the wide range of proteins, preparing nanoparticles from albumin represent an attractive strategy. Albumin is easily commercially available, it is obtained with significant quantities from bovine serum (BSA), human serum (HSA), egg white (OVA), and from soybean, milk, and grains. In addition, it is known that a significant amount of drug can be incorporated into albumin particle matrix because of the different drug binding sites present in the albumin molecule. Moreover, albumin nanoparticles could allow the electrostatic adsorption of negatively and positively charged molecules without the requirement of other compounds due to the defined primary structure of albumin protein, and its high content of charged amino acids [63].

\section{Albumin interaction with NE and SLPI}

Despite the lack of research about the interaction of albumin with both NE and SLPI and its effect on their activity, some evidence can be collected from the literature concerning this topic. in a study made on the interaction of human neutrophil elastase with soluble and insoluble protein substrates, including BSA, it was found that BSA can rapidly form a reversible complex with NE as an efficient substrate. This complex was shown to be reversibly dissociated in the presence of other factor such as eglin $\mathrm{C}$ at low concentration [64].

However, in a more recent study, NE was incubated with two antibiotics colistin and tobramycin in the presence and absence of BSA. It was found that these two antibiotics have an inhibition effect on the activity of elastase. However, in the presence of albumin, the activity of NE was significantly elevated. Suggesting that albumin has an activating effect on NE, or at least a strong dependency of NE activity on albumin [63]. These findings even if they did not give a profound explanation about the NE-albumin relationship, it can indicate that if an interaction is present, it is a non-destructive interaction between these two molecules.

As for SLPI, a study investigated the binding of SLPI to immunoglobulins (Ig) and BSA. This study showed that SLPI can interact with Ig but not with BSA in a medium with $10 \mathrm{mM}$ phosphate buffer saline ( $\mathrm{pH}$ 7.5) [64].

\section{A new strategy of the preparation of NE and SLPI loaded albumin nanoparticles}

Based on the above, a new strategy for NE and SLPI delivery and targeting could be suggested. Albumin based nanoparticles could be prepared via different method to carry these to molecules separated. One of the suggested preparation methods is the anti-solvent precipitation (also known as nanoprecipitation or solvent displacement method). In these methods, albumin and the molecule of interest can be dissolved in a solvent phase. At pH 7, albumin molecules should be negatively charged, and NE and SLPI should be positively charged. A non-solvent phase that is miscible with the solvent will be added rapidly under stirring. This will cause a supersaturation of protein molecule to thermodynamic instability. The system will then regain its stability by reducing the dissolved protein concentration in the form of particles. A portion of these proteins that are collapsed into particles should entrap NE or SLPI. By applying this protocol, Elastase loaded, or SLPI loaded albumin nanoparticles should be obtained (Figure 6).

These formulations could in theory be prepared successfully and can be applied as therapeutic agents for different kinds of situation. NEloaded albumin nanoparticles can be applied as antibacterial agents in the same way as mentioned in (Figure 4). By encapsulating elastase with certain surface modifications on the particles surface, NE can act exclusively on bacteria killing and therefore increasing its desired activity and reducing and perhaps eliminating its undesired proteolytic effect against biological tissues. This will result into an efficient antibacterial killing strategy with negligible side effects.

On the other hand, SLPI-loaded nanoparticles can be used as a NE inhibiting agent to treat acute lung injury, COPD, and other lung related diseases where NE plays a major role. By encapsulating SLPI, this concept can focus and increase the activity of SLPI against NE. in addition, as mentioned above, SLPI has an important anti-bacterial activity. By using this strategy, particles loaded with SLPI can be used as antibacterial agent to specifically target bacteria in vivo. This may also be a good alternative for antibiotic treatment that started to lose its efficiency.

However, to adopt such strategy, different steps of optimization and characterization should be completed. The optimization of nanoparticles is basically obtaining a desired shape, size, and charge for in vivo administration. These characteristics can vary according to the route of administration to be used. These physico-chemical properties of nanoparticles can be easily manipulated by controlling the initial parameters of the nanoprecipitation method. In addition, different kinds of tests should be completed, the encapsulation efficiency and the drug release profile should be highlighted, as for the stability of

Table 1. Protein-based nanoparticles and their encapsulated products

\begin{tabular}{|c|c|c|c|}
\hline Protein & Encapsulated product & Aim of the study & Reference \\
\hline Zein & $\begin{array}{c}\text { Dihydroxycoumarin } \\
\text { Quercetin } \\
\text { Curcumin }\end{array}$ & $\begin{array}{l}\text { Characterization } \\
\text { Stability } \\
\text { Mucoadheion study }\end{array}$ & $\begin{array}{l}{[61]} \\
{[62]} \\
{[63]}\end{array}$ \\
\hline Gelatin & $\begin{array}{c}\text { Tizanidine hydrochloride, } \\
\text { Gatifloxacin } \\
\text { Fluconazole }\end{array}$ & Drug release profile & {$[64]$} \\
\hline BSA & Bone morphogenetic protein 2 & Bioactivity & {$[65]$} \\
\hline HSA & $\begin{array}{l}\text { Gd-DTPA } \\
\text { Paclitaxel }\end{array}$ & $\begin{array}{l}\text { Carcinoma imaging } \\
\text { Breast cancer chemotherapy }\end{array}$ & $\begin{array}{l}{[66]} \\
{[67]}\end{array}$ \\
\hline Soy protein isolates & Vitamin B12 & $\begin{array}{l}\text { Improve intestinal transport and absorption } \\
\text { of B12 }\end{array}$ & {$[68]$} \\
\hline Silk & $\begin{array}{l}\text { Alcain blue } \\
\text { Rhodamine B } \\
\text { insulin }\end{array}$ & $\begin{array}{l}\text { In-vitro release profile } \\
\text { In-vitro stability }\end{array}$ & $\begin{array}{l}{[69]} \\
{[70]}\end{array}$ \\
\hline
\end{tabular}



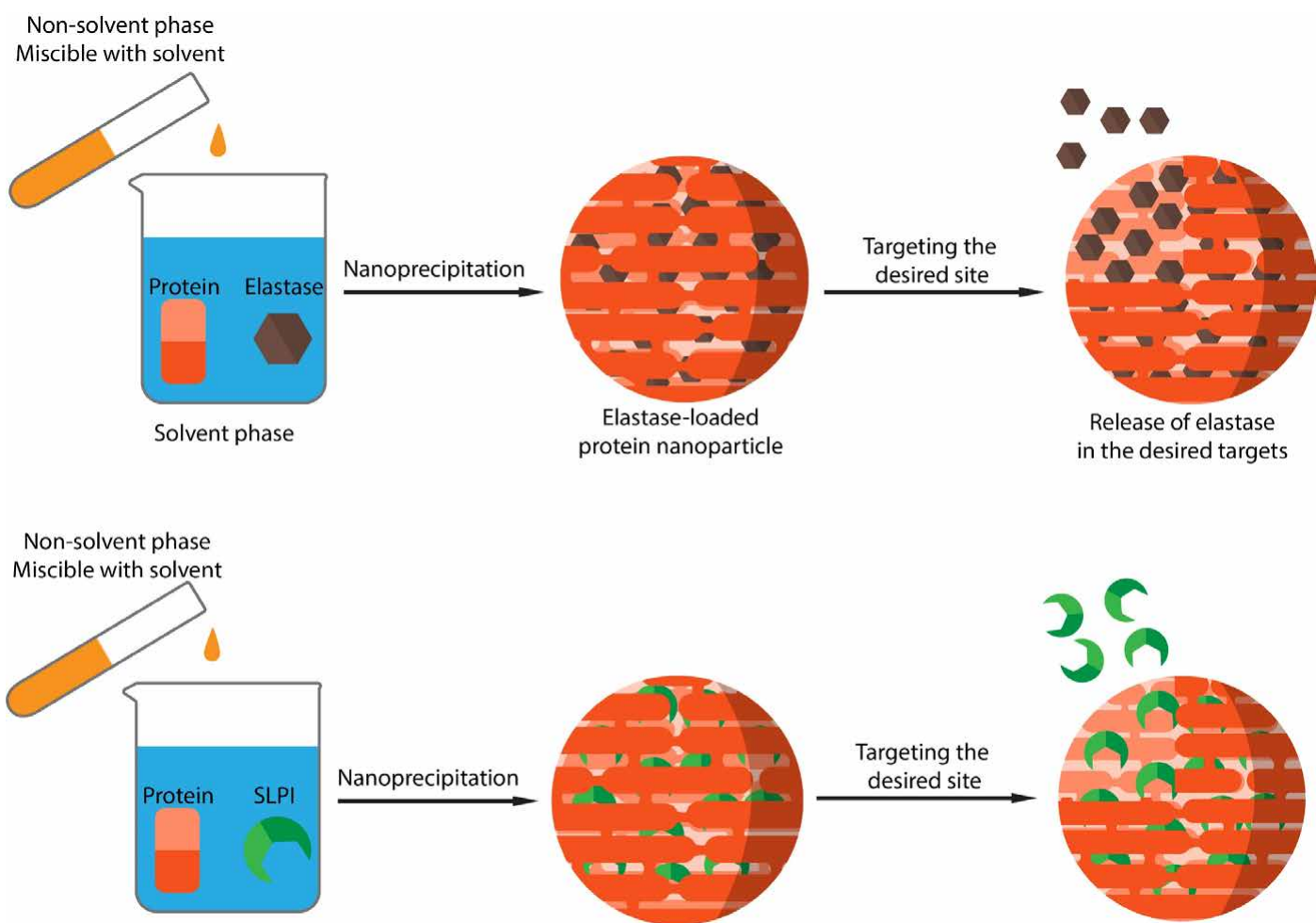

Solvent phase

SLPI-loaded protein nanoparticle

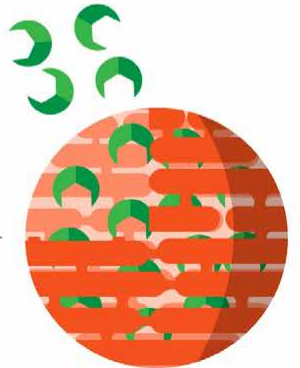

Release of SLPI in the desired targets

Figure 6. preparation of NE loaded, and elastase loaded albumin nanoparticles via nanoprecipitation method

nanoparticles. In vitro testing should also be completed to identify the effect of albumin particles on the activity of NE and SLPI. And finally, in vivo testing is required to understand the behaviour of these formulations in circulation.

\section{Conclusion}

Human neutrophil elastase is an important enzyme with a main role in neutrophil related antibacterial killing, however, due to its proteolytic activity, it can be the cause to tissue degradation in different acute lung injuries and diseases. SLPI is a natural small protein that has an important inhibition activity on NE and an antibacterial activity. The imbalance in NE/SLPI ratio in lungs is the main reason for lung tissue destruction. A new strategy was suggested to make use of these molecules for treatment of infection and acute injury. Albumin based nanoparticles can be used as drug carriers for these molecules into the site of action to focus the activity and eliminate the side effects. However, due to the lack of evidence, this suggestion should be tested and optimized to be applied as an efficient strategy in NE and SLPI related actions.

\section{Conflicts of interest}

The authors declare no conflicts of interest.

\section{References}

1. Pham CTN (2006) Neutrophil serine proteases: Specific regulators of inflammation. nature reviews Immunology 6: 541-500.

2. Faurschou M and Borregaard N (2003) Neutrophil granules and secretory vesicles in inflammation. Microbes and Infection 5: 1317-1327. [Crossref]

3. Korkmaz B, Moreau T and Gauthier F (2008) Neutrophil elastase, proteinase 3 and cathepsin G: Physicochemical properties, activity and physiopathological functions. Biochimie 90: 227-242.
4. Dai R (2017) Neutrophils and neutrophil serine proteases are increased in the spleens of estrogentreated C57BL/6 mice and several strains of spontaneous lupus-prone mice. PLOS ONE 12: 1-19.

5. PHAM C (2008) Neutrophil serine proteases fine-tune the inflammatory response. The International Journal of Biochemistry and Cell Biology 40: 1317-1333.

6. Gullberg U (1997) Biosynthesis, processing and sorting of neutrophil proteins: insight into neutrophil granule development. European journal of haematology 58: 137-153.

7. Korkmaz B (2010) Neutrophil elastase, proteinase 3, and cathepsin G as therapeutic targets in human diseases. Pharmacological reviews 62: 726-759.

8. Hansen G (2011) Unexpected active-site flexibility in the structure of human neutrophil elastase in complex with a new dihydropyrimidone inhibitor. Journal of Molecular Biology Elsevier Ltd 409: 681-691.

9. Bode W (1986) X-ray crystal structure of the complex of human leukocyte elastase (PMN elastase) and the third domain of the turkey ovomucoid inhibitor. The EMBO journal. European Molecular Biology Organization 5: 2453.

10. Koehl C, Knight CG, Bieth JG (2003) Compared action of neutrophil proteinase 3 and elastase on model substrates. Favorable effect of S-P interactions on proteinase 3 catalysts. Journal of Biological Chemistry ASBMB 278: 12609-12612.

11. Tanaka T (1985) Human leukocyte cathepsin G. Subsite mapping with 4-nitroanilides, chemical modification, and effect of possible cofactors. Biochemistry. ACS Publications 24: 2040-2047.

12. Kettritz R (2016) Neutral serine proteases of neutrophils. Immunological Reviews 273: 232-248.

13. van Kesse KPM, Bestebroer J, van Strijp JAG (2014) Neutrophil-mediated phagocytosis of Staphylococcus aureus. Frontiers in Immunology 5: 1-12.

14. Nauseef WM (2014) Proteases, neutrophils, and periodontitis: The NET effect. Journal of Clinical Investigation 124: 4237-4239.

15. Weinrauch Y (2002) Neutrophil elastase targets virulence factors of enterobacteria Nature 417: 91-94.

16. Belaaouaj A (2000) Degradation of outer membrane protein a in Escherichia coli killing by neutrophil elastase. Science 289: 1185-1187. 
17. Odeberg H, Olsson I (1976) Microbicidal mechanisms of human granulocytes: synergistic effects of granulocyte elastase and myeloperoxidase or chymotrypsin-like cationic protein. Infection and immunity 14: 1276-1283.

18. Brinkmann V (2004) Neutrophil Extracellular Traps Kill Bacteria. Science 303: 15321535.

19. Martinez NE (2017) Tetrahydroisoquinolines: New Inhibitors of Neutrophil Extracellular Trap (NET) Formation. Chem Bio Chem 18: 888-893. [Crossref]

20. Papayannopoulos V (2010) Neutrophil elastase and myeloperoxidase regulate the formation of neutrophil extracellular traps. Journal of Cell Biology 191: 677-691.

21. Sørensen OE (2014) Papillon-Lefèvre syndrome patient reveals species-dependent requirements for neutrophil defenses. Journal of Clinical Investigation 124: 4539-4548.

22. Korkmaz B, Moreau T, Gauthier F (2008) Neutrophil elastase, proteinase 3 and cathepsin G: Physicochemical properties, activity and physiopathological functions. Biochimie 90: 227-242.

23. Alfaidi M (2015) Neutrophil elastase promotes interleukin-1 $\beta$ secretion from human coronary endothelium. Journal of Biological Chemistry 290: 24067-24078.

24. Macleod T (2016) Neutrophil Elastase-mediated proteolysis activates the antiinflammatory cytokine IL-36 Receptor antagonist. Scientific Reports. Nature Publishing Group 6: 24880.

25. Voynow JA (2004) Neutrophil elastase induces mucus cell metaplasia in mouse lung. American journal of physiology. Lung cellular and molecular physiology 287: L1293302.

26. Nadel JA (2007) Innate immune mucin production via epithelial cell surface signaling: relationship to allergic disease. Current opinion in allergy and clinical immunology 7: 57-62.

27. Meyer-Hoffert U, Wingertszahn J, Wiedow O (2004) Human Leukocyte Elastase Induces Keratinocyte Proliferation by Epidermal Growth Factor Receptor Activation. Journal of Investigative Dermatology 123: 338-345.

28. Taipale J (1995) Human mast cell chymase and leukocyte elastase release latent transforming growth factor-beta 1 from the extracellular matrix of cultured human epithelial and endothelial cells. The Journal of biological chemistry 270: 4689-4696.

29. Voynow j, fischer b, zheng s (2008) Proteases and cystic fibrosis. The International Journal of Biochemistry and Cell Biology 40: 1238-1245. [Crossref]

30. Quinn D (2010) Antiproteases as Therapeutics to Target Inflammation in Cystic Fibrosis. The Open Respiratory Medicine Journal 4: 20-31.

31. Demedts IK (2006) Role of apoptosis in the pathogenesis of COPD and pulmonary emphysema. Respiratory Research 7: 53. [Crossref]

32. Lee KH (2015) Neutrophil elastase differentially regulates IL-8 and VEGF production by cigarette smoke extract. Journal of Biological Chemistry 290: :28438-28445.

33. Thaikoottathil JV (2009) Cigarette smoke extract reduces VEGF in primary human airway epithelial cells. European Respiratory Journal 33: 835-843.

34. Epstein FH and Weiss SJ (1989) Tissue Destruction by Neutrophils. New England Journal of Medicine 320: 365-376. [Crossref]

35. Morrison HM (1990) Inhibition of Human Leukocyte Elastase Bound to Elastin: Relative Ineffectiveness and Two Mechanisms of Inhibitory Activity. American Journal of Respiratory Cell and Molecular Biology 2: 263-269.

36. Boxio R (2016) Neutrophil elastase cleaves epithelial cadherin in acutely injured lung epithelium. Respiratory Research. Respiratory Research 17: 129.

37. Moroy G (2012) Neutrophil Elastase as a Target in Lung Cancer. Anti-Cancer Agents in Medicinal Chemistry 12: 565-579. [Crossref]

38. Si-Tahar M (2000) Constitutive and regulated secretion of secretory leukocyte proteinase inhibitor by human intestinal epithelial cells. Gastroenterology 118: 10611071.

39. Koizumi M (2008) Complex of human neutrophil elastase with 1/2SLPI. Journal of Synchrotron Radiation 15: 308-311. [Crossref]

40. Boudier C and Bieth JG (1989) Mucus proteinase inhibitor: a fast-acting inhibitor of leucocyte elastase. Biochimica et biophysica acta 995 : 36-41.

41. Lee CH (1993) Distribution of Secretory Leukoprotease Inhibitor in the Human Nasal Airway. American Review of Respiratory Disease 147: 710-716.

42. Rice W, Weiss S (1990) Regulation of proteolysis at the neutrophil-substrate interface by secretory leukoprotease inhibitor. Science 249: 178-181.
43. Zabieglo K (2015) The inhibitory effect of secretory leukocyte protease inhibitor (SLPI) on formation of neutrophil extracellular traps. J Leukoc Biol 98: 99-106.

44. Moreau T (2008) Multifaceted roles of human elafin and secretory leukocyte proteinase inhibitor (SLPI), two serine protease inhibitors of the chelonianin family. Biochimie 90: 284-295.

45. Majchrzak-Gorecka M (2016) Secretory leukocyte protease inhibitor (SLPI), a multifunctional protein in the host defense response. Cytokine and Growth Factor Reviews. Elsevier Ltd 28: 79-93.

46. Antoniades CG (2014) Secretory leukocyte protease inhibitor: A pivotal mediator of anti-inflammatory responses in acetaminophen-induced acute liver failure. Hepatology 59: $1564-1576$.

47. Taggart CC (2002) Secretory leucoprotease inhibitor prevents lipopolysaccharideinduced I $\mathrm{B} \mathrm{B} \alpha$ degradation without affecting phosphorylation or ubiquitination. Journal of Biological Chemistry 277: 33648-33653.

48. Taggart CC (2005) Secretory leucoprotease inhibitor binds to NF-kappaB binding sites in monocytes and inhibits p65 binding. The Journal of experimental medicine 202: 1659-1668.

49. Hiemstra PS (1996) Antibacterial activity of antileukoprotease. Infection and immunity 64: 4520-4524. [Crossref]

50. Miller KW (1989) Secretory leukocyte protease inhibitor binding to mRNA and DNA as a possible cause of toxicity to Escherichia coli. Journal of Bacteriology 171: 2166-2172.

51. Scott A, Weldon S, Taggart CC (2011) SLPI and elafin: multifunctional antiproteases of the WFDC family. Biochemical Society Transactions 39: 1437-1440.

52. McNeely TB (1997) Inhibition of human immunodeficiency virus type 1 infectivity by secretory leukocyte protease inhibitor occurs prior to viral reverse transcription. Blood 90: 1141-1149.

53. Pillay K (2001) Secretory leukocyte protease inhibitor in vaginal fluids and perinatal human immunodeficiency virus Type 1 transmission. The Journal of Infectious Diseases 183: 653-656.

54. Pandey KC, De S, Mishra PK (2017) Role of proteases in chronic obstructive pulmonary disease. Frontiers in pharmacology 8: 512.

55. Groutas WC, Dou D, Alliston KR (2011) Neutrophil elastase inhibitors. Expert Opinion on Therapeutic Patents 21:339-354.

56. Guarnieri F (2010) A human surfactant peptide-elastase inhibitor construct as a treatment for emphysema. Proceedings of the National Academy of Sciences 107 10661-10666.

57. Torchilin VP (2000) Drug targeting. European Journal of Pharmaceutical Sciences 11: S81-S91.

58. Trierweiler LF, Trierweiler JO (2011) industrial production of polymeric nanoparticles: alternatives and economic analysis. in nanocosmetics and nanomedicines: New Approaches for Skin Care 18: 123-138.

59. Govender T (1999) PLGA nanoparticles prepared by nanoprecipitation: Drug loading and release studies of a water-soluble drug. Journal of Controlled Release 57: 171-185.

60. Martínez Rivas CJ (2017) Nanoprecipitation process: From encapsulation to drug delivery. International Journal of Pharmaceutics. Elsevier BV 532: 66-81.

61. Elzoghby AO (2012) Albumin-based nanoparticles as potential controlled release drug delivery systems. Journal of controlled release: official journal of the Controlled Release Society. Elsevier BV 157: 168-182

62. Baici A (1990) Interaction of human leukocyte elastase with soluble and insoluble protein substrates. A practical kinetic approach. Biochimica et Biophysica Acta (BBA)/ Protein Structure and Molecular 1040: 355-364.

63. Hector A, Kappler M, Griese M (2010) In vitro inhibition of neutrophil elastase activity by inhaled anti-pseudomonas antibiotics used in cystic fibrosis patients. Mediators of Inflammation 2: 1-5.

64. Kamada M (1999) Binding of human secretory leukocyte protease inhibitor in uterine cervical mucus to immunoglobulins: Pathophysiology in immunologic infertility and local immune defense. Fertility and Sterility 71: 1108-1114.

Copyright: (C)2018 Tarhini $\mathrm{M}$. This is an open-access article distributed under the terms of the Creative Commons Attribution License, which permits unrestricted use, distribution, and reproduction in any medium, provided the original author and source are credited. 